\title{
Growth Cone Behavior Underlying the Development of Stereotypic Synaptic Connections in Drosophila Embryos
}

\author{
Marnie E. Halpern, ${ }^{a}$ Akira Chiba, Jørgen Johansen, ${ }^{\mathrm{b}}$ and Haig Keshishian \\ Department of Biology, Yale University, New Haven, Connecticut 06511
}

\begin{abstract}
Each muscle fiber in the segmented body wall of Drosophila larvae is innervated by anatomically stereotyped neuromuscular junctions. These synapses arise through the selective choices of motoneuronal growth cones at their peripheral targets. Using digital optical microscopy of staged intracellular dye fills, we have singly identified embryonic motoneurons and have examined individual growth cones when they contact and differentiate at the target cells. There is a precise connectivity between motoneuron and muscle fiber, which is the direct consequence of growth cone behavior. We have also found that Drosophila muscle fibers possess molecularly heterogeneous cell surfaces that may be involved in growth cone recognition of appropriate targets. Fasciclin III, a homophilic adhesion molecule, is coexpressed by several of the efferent growth cones and in a site-specific fashion by the target muscle fiber's membrane. The fasciclin III expression is transient, corresponding to the period in embryogenesis when the first neuromuscular contacts are made. Upon encountering the target cell surface, the growth cones can sprout stereotypically arrayed filopodial processes, orient along the anterior-posterior axis, and turn in predictable directions. Subsequently, terminal branches are established in a nonrandom order. These phenomena were found to occur in two motoneurons that innervate adjacent muscle fiber targets, and may be general features of neuromuscular synaptogenesis in Drosophila.
\end{abstract}

How the exploring tips of embryonic neurons recognize their target cells and evolve from growth cones into synapses remains a major question of developmental neurobiology. Studies addressing the selective fasciculation and pathway choices made during early axon outgrowth have demonstrated that these events can be highly predictable in both vertebrates (Landmesser, 1980; Eisen et al., 1986; Kuwada, 1986) and invertebrates (Bentley

\footnotetext{
Received Mar. 6, 1991; revísed May 2, 1991; accepted May 13, 1991.

We thank S. Benzer for the $22 \mathrm{C} 10$ antibody, P. Taghert for the Lucifer yellow antiserum, T. Elkins and C. Goodman for the fasciclin III antibody, and M. Levine for the even-skipped antiserum. We also thank C. Goodman and N. Patel for helpful discussions, R. J. Wyman, W. McGinnis, M. S. Anderson, and E. Harkins for their comments on the manuscript, and T. Chang for the use of material. This work was supported by an NIMH predoctoral fellowship to M.E.H., an NIH training grant postdoctoral fellowship to J.J., and grants from the NIH and March of Dimes Birth Defects Foundation to H.K.

Correspondence should be addressed to Haig Keshishian, Department of Biology, Yale University, P.O. Box 6666 KBT, New Haven, CT 06511.

a Present address: Institute of Neuroscience, 219 Science III, University of Oregon, Eugene, OR 97403.

Present address: Zoology Department, Science Building II, Iowa State University, Ames, IA 50011.

Copyright (C) 1991 Society for Neuroscience $0270-6474 / 91 / 113227-12 \$ 05.00 / 0$
}

and Keshishian, 1982; Bentley and Caudy, 1983; Raper et al., 1983; Goodman et al., 1984; Bastiani et al., 1987). The biochemical nature of some of the presumptive guidance or adhesion cues involved in axonogenesis has also been examined (reviewed by Anderson, 1988; Dodd et al., 1988; Harrelson and Goodman, 1988; Jessell, 1988). Although we have a better understanding of the mechanisms of axon guidance, there is still much to learn about how a navigating growth cone distinguishes its appropriate target, and the cellular events that ensue.

Drosophila is a favorable organism for examining the cellular and biochemical processes underlying the development of neural connectivity. The embryonic and larval neuromuscular junctions constitute a simple and acccssible array of synapses (Johansen et al., 1989a). The body wall muscle fibers are constant in pattern among embryos and larvae. The initial efferent projections are also stereotypic and differentiate into motor endings of predictable shape and transmitter type (Anderson et al., 1988; Halpern et al., 1988; Johansen et al., 1989b).

In Drosophila embryos and larvae, the skeletal muscle fibers are arranged in bilaterally symmetrical, segment-specific patterns (Hertweck, 1931; Crossley, 1978; Campos-Ortega and Hartenstein, 1985). The second through seventh abdominal hemisegments (A2-A7) share a common pattern consisting of only 30 muscle fibers. Each fiber is a single multinucleate cell, recognizable by its size, polarity of insertion, and relative position in the pattern. Twelve dorsal muscle fibers are innervated via the intersegmental nerve (ISN), while the remaining fibers are innervated by four branches of the segmental nerve (SNad). During mid to late embryogenesis, $6 \mathrm{hr}$ lapse from the time the first efferent axons extend toward the periphery until functional neuromuscular connections are formed (Johansen et al., 1989 b). Anatomical stereotypy is evident in the embryonic endings when they are labeled en masse with anti-HRP (Johansen et al., 1989b).

While the studies by Johansen et al. (1989a,b) have revealed the structural stereotypy of Drosophila neuromuscular junctions, several important questions remain unanswered. Since neuron-specific antibodies label the entire complement of motoneurons contacting a muscle fiber, it is difficult to distinguish how individual growth cones behave as they make their projections. For example, it is not known how the specific ending morphology arises during development, nor the extent to which these endings are subject to remodeling. Key to understanding these events is the identification of individual motoneuronal growth cones. Drosophila neurons with efferent projections have been described previously (Thomas et al., 1984; Jacobs and Goodman, 1989; Halpern and Keshishian, 1990), and recently Sink and Whitington (1991) have characterized the peripheral targets of abdominal motoneurons. 
The high degree of stereotypy evident in Drosophila neuromuscular endings provides a unique opportunity to examine how precision in synaptic morphology is established as a function of growth cone behavior. In Drosophila, neuromuscular development proceeds toward a predictable outcome, where each muscle fiber will ultimately bear its own morphologically and neurochemically characteristic synapses. Examining how individual growth cones generate these synapses will provide important insight into the mechanisms that control specific neuronal form.

To understand how structurally characteristic synapses arise, we have identified the targets and studied in detail the development of two cells, the neurons RPl and RP3 (Patel et al., 1987; Jacobs and Goodman, 1989). These motoneurons lie adjacent to each other in the CNS, grow through the CNS by following a similar trajectory, and exit via the same nerve bundle to innervate adjacent muscle fibers. We have used computerenhanced digital optical microscopy to image unfixed, intracellularly labeled neurons and have reconstructed the events during the target muscle fiber exploration in situ in a series of staged embryonic dye fills. Motoneurons in the embryo target not only specific muscle fibers but characteristic domains of the target cell's membrane. The growth cones subsequently generate the characteristic neuromuscular endings through a sequence of predictable projections on the target surface.

These results were previously reported in part in an abstract (Halpern and Keshishian, 1990).

\section{Materials and Methods}

Embryo preparations. Embryos of desired stages were obtained from population cages of wild-type Drosophila melanogaster Canton S flies, maintained on apple juice agar plates at $25^{\circ} \mathrm{C}$. Eggs collected during a $1 \mathrm{hr}$ period were allowed to develop at $25^{\circ} \mathrm{C}$ until appropriate stages were reached. Embryos were staged using morphological criteria (Campos-Ortega and Hartenstein, 1985; Johansen et al., 1989b). In this study, the estimated age of the embryos in hours as well as the morphological stage are given. To calibrate the staging, in several dye-filled preparations the state of neuromuscular development was confirmed independently by anti-HRP labeling all neurons (Johansen et al., 1989b). To prepare fillets for dye filling, embryos were dechorionated manually and transferred to a sliver of double-sided lape within a well made of silicon sealant on a glass slide. After adequate numbers of embryos were lined up on the tape, either osmotically balanced insect saline (Johansen et al., 1989a) or a modified Schneider insect medium (GIBCO) was added to the well. In this medium, growth cones will elongate and project filopodia on the surfaces of embryonic fillets (Chiba et al., 1990). Embryos were devitellinized using glass micropipettes and stuck dorsal side up to a clean surface on the slide. Internal organs were removed through a dorsal incision. The body wall was opened and stretched flat to reveal the CNS and peripheral nerves. We found that 17 -hr or older embryos (stage 17) stick well to glass coated with a thin layer of tacky Sylgard silicone clastomer (Dow Corning). Prior to dye filling, fresh aerated saline or Schneider medium was added.

Intracellular labeling. Living cells were observed using a long-working-distance $40 \times 0.75 \mathrm{NA}$ water-immersion objective and differential interference contrast (Nomarski) optics. Images were relayed to a highresolution video camera via a $5 \times$ ocular and displayed on the monitor. With this method, neuronal cell bodies are revealed with detail and clarity, simplifying their identification and impalement. Neurons in abdominal segments were dye filled using 250-600 M electrodes with tips containing 10\% Lucifer yellow in water and shanks backfilled with $1 \mathrm{M} \mathrm{LiCl}$. Dye was passed by a $0.1-0.2 \mathrm{nA}$ continuous hyperpolarizing current until motoneurons were filled to their terminal arborizations.

We observed changes in the morphology of both growth cones, filopodia, and developing muscle fibers after paraformaldehyde fixation. The fixation-induced changes were observed at all stages studied. For this reason, all the dye fills in this study were characterized in living, unfixed preparations as soon as practical after dye filling. We recon- structed the behavior of the growth cones by examining a series of staged dye fills in the embryos. The growth cones of 69 dye fills were analyzed in this study.

Digital optical microscopy. Fluorescent dye-filled growth cones were imaged on the monitor at high magnification using computer-enhanced digital microscopy. Images of epiilluminated cells were acquired using a silicon intensifier target video camera. Video signals were digitized by a $1024 \times 1024$ pixel image processing board with 12 bit depth in a 486 desktop computer and displayed on high-resolution video monitors as $512 \times 480$ pixel images with 8 -bit depth. The acquired images were linearly averaged for up to 32 frames to boost signal to noise and recalculated to optimize contrast. As neuronal projections are often in several focal planes, complete axonal trajectories were reconstructed by tableting the in-focus regions from several images. Final composites were convolved with a $7 \times 7$ kernel sharpening algorithm and a median filter and were stored digitally on a high-density laser optical disk drive or as analog images on a laser optical memory disk recorder. Pseudocolored composite images were photographed either from the video monitor or with a film recorder using Kodak T-MAX 100 or EKTAR 125 film.

For several embryos, the cell body locations of the RP1 and RP3 neurons were ascertained by comparing Lucifer yellow dye fills to the Nomarski image using a laser confocal microscope (Bio-Rad MRC 500). This allowed us to determine accurately whether a specific neuronal cell body corresponded to either RP1 or the deeper-located RP3.

Structural resolution of filopodia. In this study, all growth cones were imaged while alive with a calculated limiting resolution of $0.3 \mu \mathrm{m}$, using fluorescence epillumination. We have defined filopodia as those processes less than $0.5 \mu \mathrm{m}$ in width and with a length of at least $1 \mu \mathrm{m}$ emerging from the body of the growth cone. The Lucifer yellow-labeled filopodia are of similar length and caliber to those observed using immunocytochemically labeled preparations (Johansen et al., 1989b), and they correspond well with motile filopodia imaged in time lapse using confocal laser microscopy of 1-1'-dioctodecyl-3,3,3', $3^{\prime}$-tetramethylindocarbocyanine perchlorate (Dil) labeled neurons (Chiba el al., 1990).

Immunocytochemistry. For double labeling of dye-filled embryos, fillets were fixed in situ for $15-30 \mathrm{~min}$ at room temperature with freshly made $4 \%$ paraformaldehyde in $100 \mathrm{~mm} \mathrm{NaH}_{2} \mathrm{PO}_{4}$, pH 7.4. The fillets were washed several times in PBS $\left(20 \mathrm{~mm} \mathrm{NaH}_{2} \mathrm{PO}_{4}, 150 \mathrm{~mm} \mathrm{NaCl}\right.$, adjusted to $\mathrm{pH} 7.2$ ) and then carefully scraped off the slide using a glass micropipette and transferred to a multiwell dish containing TBS (PBS with $0.3 \%$ Triton $\mathrm{X}-100$ ). Tissues were blocked for $30 \mathrm{~min}$ in $1 \%$ BSA in TBS, and incubations of the primary antibodies were performed overnight at $4^{\circ} \mathrm{C}$ at the appropriate concentrations in TBS (anti-HRP, Johansen et al., 1989b; anti-Lucifer yellow, Taghert et al., 1982; 22C10 monoclonal antibody, Fujita et al., 1982; fasciclin III monoclonal antibody, 2D5, Patel et al., 1987; or even-skipped antiserum, Frasch et al., 1987). Following several TBS washes, fluorescent secondary antibodies (Cappel) were used routinely at concentrations of 1:250 for $2 \mathrm{hr}$ at room temperature. The fillets were washed in PBS and water and mounted on slides in 5\% $n$-propyl gallate in glycerol (Giloh and Sedat, 1982). For peroxidase cytochemistry, incubations with the anti-Lucifer yellow rabbit serum and the fasciclin III mouse monoclonal antibody were carried out simultaneously, as were the subsequent steps for their visualization using species-specific HRP-conjugated secondary IgGs (goat anti-mouse, Jackson Immunoresearch Labs; goat anti-rabbit, Cappel). The fasciclin III monoclonal antibody $(1: 2.5$ dilution) was also combined with even-skipped antiserum (1:2500 dilution) and visualized with the appropriate corresponding fluorescently labeled secondary antibodies (sheep anti-mouse tetramethylrhodamine isothiocyanate $\mathrm{IgG}$, and goat anti-rabbit fluorescein isothiocyanate $\mathrm{IgG}, 1: 250$ dilutions, Cappel).

\section{Results}

The segmental nerve branch $b(S N b)$ develops through stereotypic axonal projections. In the larval body wall, each muscle fiber exhibits an anatomically predictable neuromuscular ending, characterized by a preferred contact site by the nerve branch, the direction and arborization of the innervating neurites, and the muscle cell surface over which synaptic boutons are placed (Johansen et al., 1989a,b). During late embryogenesis, these features arise through a sequence of growth cone-mediated tra- 
jectories. This sequence is illustrated for the $\mathrm{SNb}$ in Figure $1 \mathrm{~A}$, where the entire complement of outgrowing axons was traced at four successive stages of development after being labeled with anti-HRP immunocytochemistry. As shown schematically in Fig. $1 B, \mathrm{SNb}$ carries axons to only six ventral muscle fibers, 28 , $7,6,14,13$, and 12 (listed in order from the ventral midline). The first SNb growth cones emerge from the CNS between 12 and $13 \mathrm{hr}$ of embryogenesis (stage 15). They project peripherally during the period from 13 to $16 \mathrm{hr}$ of development (stage 16), exclusively over the surfaces of already differentiated muscle fibers, sandwiched between the external and internal muscle layers (Fig. 1 $A$ ). SNb subbranches are established through the ordered divergence of different axonal growth cones at specific sites on the six target fibers. Subsequently, each contact evolves into the junctional morphology associated with the mature larval muscle fiber (Fig. $1 A$, bottom trace). The embryonic neuromuscular contacts have predictable lengths, directions, and branching patterns that resemble in many respects those seen in the third instar larva.

Characterization of the RP neurons and their targets. Each larval muscle fiber may be innervated by multiple motoneurons, as deduced from electrophysiology (Jan and Jan, 1976), neurotransmitter immunocytochemistry (Halpern et al., 1988; Johansen et al., 1989a), and electron microscopy (M. E. Halpern and W. Costello, unpublished observations). By intracellular dye filling in the CNS of unfixed, dissected embryonic fillets, we have been able to identify motoneurons and their target muscle fibers and to reconstruct the behavior of their growth cones as they differentiate into synapses.

Among the prominent cell bodies visible on the dorsal aspect of the CNS are the two paired and medially located neurons RP1 and RP3. RPI's cell body is situated between the anterior and posterior commissures, in the dorsalmost layer of cells beneath the glial sheath. RP3's cell body is located directly ventral to RP1 (Patel et al., 1987; Jacobs and Goodman, 1989). Both neurons migrate to their midline destinations from more lateral positions, beginning at $10 \mathrm{hr}$ (stage 13) of development (Patel et al., 1987; Jacobs and Goodman, 1989). The axons of both neurons project within the anterior commissure to join the contralateral ISN tract (Jacobs and Goodman, 1989).

Sink and Whitington (1991) have described the muscle fiber targets of the neurons, indicating that RP1 projects to muscle fiber 13 and that RP3 projects to muscle fibers 6 and 7 . We have confirmed this characterization as representing the predominant pattern in our dye fills, as observed in both Canton $\mathrm{S}$ and Oregon $\mathrm{R}$ wild-type flies. However, we have observed dye fills in our own data set where the projections are reversed, as confirmed by dual channel confocal microscopy using both Lucifer yellow fluorescence and Nomarski optics (RP1, $n=3$; $\mathrm{RP} 3, n=2$ ). This may represent reversed cell body positions as a consequence of the RP cell migrations.

Figure $2 A$ shows a computer-enhanced image of the earliest stage in growth cone differentiation of RP3 observed on muscle fibers 6 and 7, shown here in a 12.5-13.5 hr (late stage 15) embryo. We analyzed by means of intracellular dye fills the differentiation of the homologous growth cones on these muscle fibers in segments from A2 to A7, through the period of 12-17 hr (stages 15-17) of development, based on 34 dye fills made in 31 embryos. The Lucifer yellow-filled growth cone (Fig. $2 B$ ) in a 14-15-hr (stage 16) embryo is shown compared to the corresponding Nomarski image of the muscle fibers in Fig. $2 C$. We found that the growth cones and mature endings always

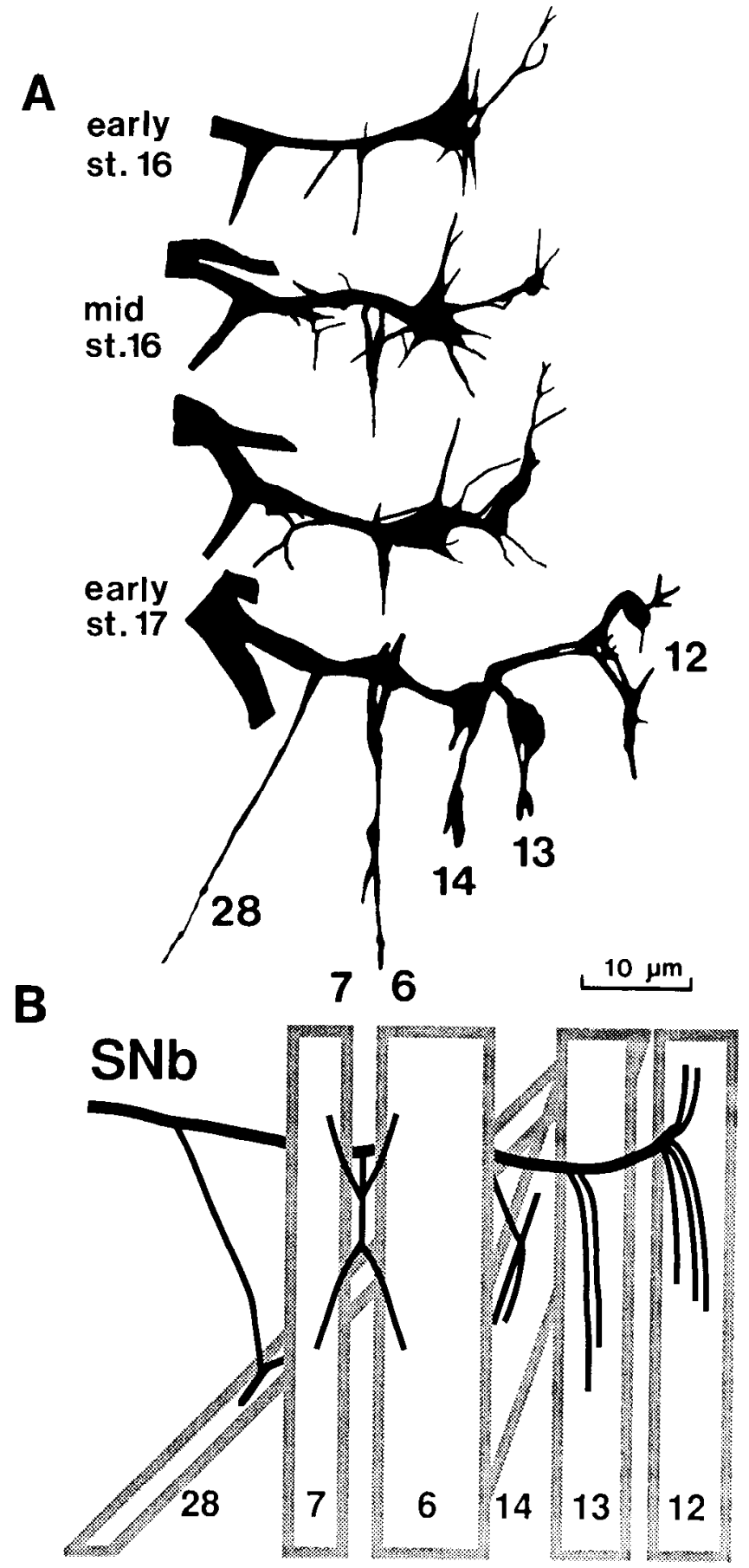

Figure 1. The six muscle fiber targets of the $\mathrm{SNb}$ are innervated in a morphologically stcrcotyped fashion. $A$, Progression of SNb development during the interval between 13 and $17 \mathrm{hr}$ of embryogenesis (stages 16-17). Growth cones are arrayed on specific membrane surfaces for each of the six target muscle fibers. From these contact sites, characteristic trajectories of predictable length and direction are made on each fiber. In the lowest drawing, each target muscle fiber is indicated by its designated number (Crossley, 1978). Tracings were made from enhanced video images of embryonic fillets labeled with anti-HRP as previously described (Johansen et al., 1989b). Scale bar refers to $A$. $B$. A schematic representation of the $\mathrm{SNb}$ nerve's neuromuscular endings as seen during the larval stages. 

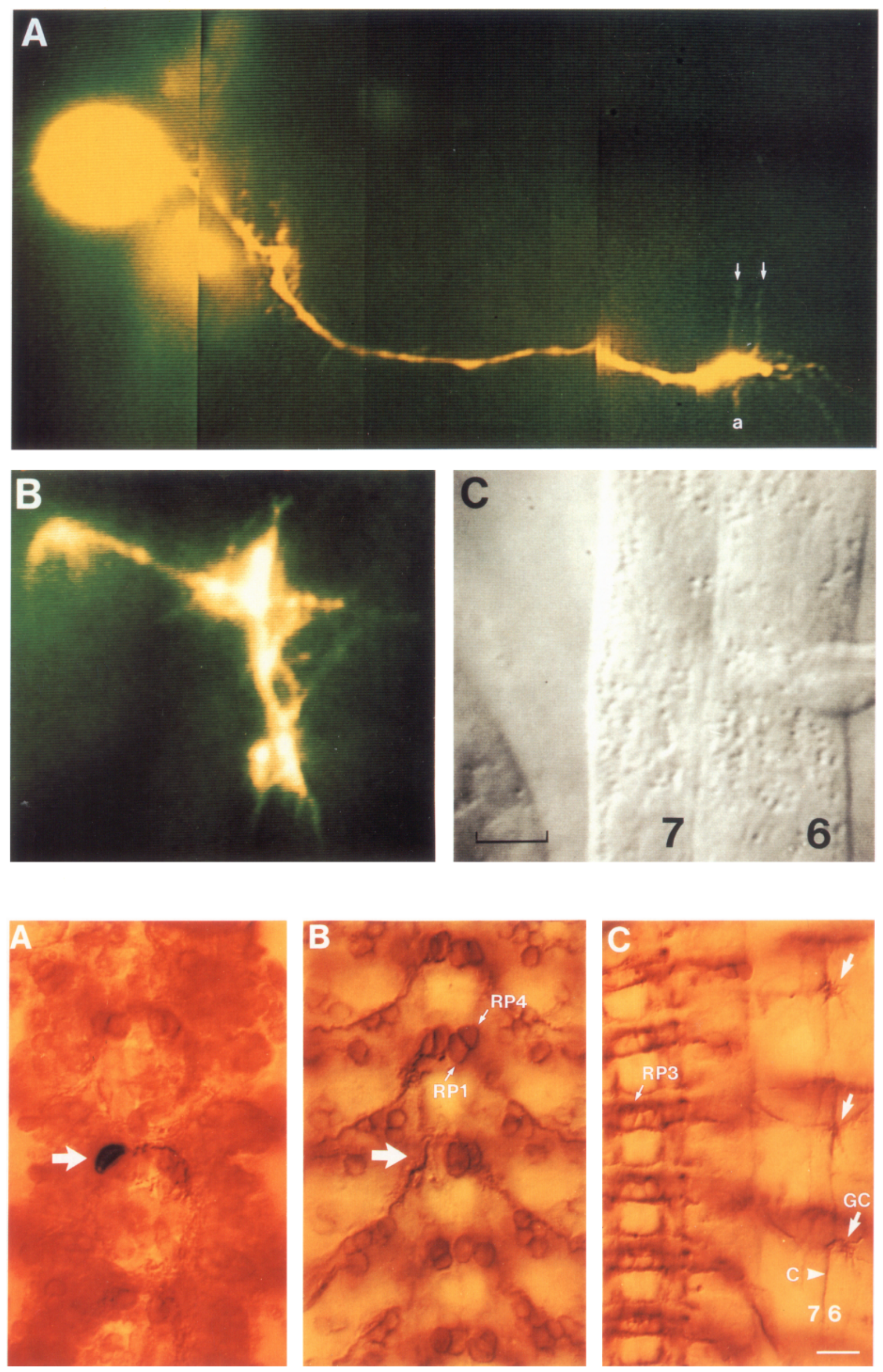
innervate equally and exclusively the two internal longitudinal muscle fibers 6 and 7.

Because of the small size of the RP cell bodies (about $4 \mu \mathrm{m}$ ) and their similar CNS trajectories (Jacobs and Goodman, 1989), we employed a number of tests to confirm their identity. Several RP neurons express fasciclin III on both their cell bodies and axons beginning at $10 \mathrm{hr}$ (stage 12-13; Patel et al., 1987). Figure $3 A$ shows a labeled contralaterally projecting neuron (in this specific dye fill the axon was traced to muscle fibers 6 and 7). The cell body is homologous by position to the fasciclin IIIrevealed RP cells in other segments. In the embryo shown in Figure $3 B$, the cell body of a Lucifer yellow dye-filled motoneuron that had projected to muscle fibers 6 and 7 was extirpated. The remaining cells were then labeled with the fasciclin III-specific antibody. Consistent with their removal in the indicated segment (arrow, Fig. $3 B$ ), only the remaining, bilaterally symmetrical RP neurons exhibit fasciclin III labeling.

In addition to RP1 and RP3, we observed a third fasciclin III-immunoreactive neuron lying immediately lateral to and usually partially overlapping dorsally RP1 in each hemisegment (Fig. 3B). To identify this neuron and the other RP cells in the vicinity, we examined the expression of both fasciclin III and even-skipped. Even-skipped is a pair rule segmentation gene expressed at this stage of development by the neuron RP2 (Doe et al., 1988), but not by the other RP cells. Figure 4 shows a double fluorescence labeling, with the two dorsal fasciclin IIIpositive cells displayed as a negative image superimposed on the positive image of the even-skipped-labeled RP2 neurons. Through dye filling ( $n=6), \mathrm{RP} 2$ was observed to extend its axon ipsilaterally, exiting the ISN to innervate dorsal targets, confirming the observation of Sink and Whitington (1991). The neurons aCC and pCC are also revealed by their even-skipped immunoreactivity. The lateral fasciclin III-immunoreactive cell corresponds to the neuron RP4 ( $n=5$ dye fills), described by Sink and Whitington (1991) as innervating muscle fiber 13.

Using fasciclin III immunocytochemistry, we have examined the RP1, RP3, and RP4 cell body locations by tracing nearly 500 neurons in 82 hemisegments from eight 15-hr (stage 16) embryos. The three RP neurons are present in each abdominal segment except A8. We observed that in $87 \%$ of the hemisegments the neuron RP4 lies partially dorsal to RP1, although the overlap rarely exceeds half the cell's diameter. In all hemiseg- ments examined, RPl's cell body is always located directly dorsal to RP3.

The RP1 growth cone makes successive choices in both the CNS and periphery as it establishes its mature axonal trajectory. We have analyzed this in detail using enhanced video microscopy of dye-filled axons double labeled with $22 \mathrm{C} 10$, a monoclonal antibody that stains subsets of neurons and fascicles in the developing nervous system, thereby revealing features of the neuropil not readily detected with Nomarski optics (Fujita et al., 1982; Canal and Ferrús, 1986; Jacobs and Goodman, 1989). The CNS trajectory of RP1 has been described by Patel et al. (1987) and Jacobs and Goodman (1989), who showed that RP1 first extends its axon anteriorly and contralaterally to pioneer a fascicle within the anterior commissure. At the longitudinal connective, the axon turns posteriorly and follows the ISN tract out of the neuropil. We found that as RPl exits the CNS its axon crosses to the segmental nerve and continues along the $\mathrm{SNb}$ branch to contact muscle fiber 13 in the next posterior segment. This behavior of crossing to $\mathrm{SNb}$ is shown by all other fasciclin III-positive axons exiting via the ISN tract, including RP3 and RP4. A diagram of the RP neurons and their trajectories is shown in Figure 5.

Both growth cones and the muscle fibers 6 and 7 express fasciclin III during their initial contact. Fasciclin III is a cell surface glycoprotein (Patel et al., 1987) that has been shown to mediate homophilic adhesion in vitro (Snow et al., 1989). Antibodies to fasciclin III reveal a transient expression on the surfaces of several SNb growth cones, including the RP neurons, as they grow over their target surfaces (present results; $A$. Chiba and $H$. Keshishian, unpublished observations). It is also expressed at the same time by muscle fibers 6 and 7 , in a site-specific fashion. The muscle staining is transient and is restricted to two sites on the muscle fiber surfaces. Fasciclin III is first expressed on the muscle fiber insertion sites (apodemes), where it is detected at 9-10 hr (stage 12-13; $n=4$ embryos). Later at $12 \mathrm{hr}$ (stage 15; $n=5$ embryos) the staining also is detected on the membrane surfaces of fibers 6 and 7 where they contact each other, which we term the "cleft" region (Fig. 3C, arrowhead labeled "C"; see also Fig. 5). It is at this site that the innervating growth cone initiates its synaptic contact by forcing its way through the cleft to reach the muscle surface facing the embryo's interior. Fasciclin III staining in the cleft is present through 14-16 hr (stage

$\longleftarrow$

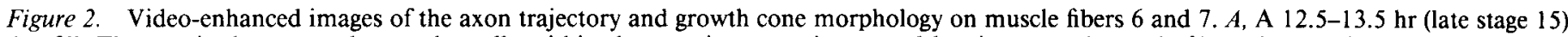

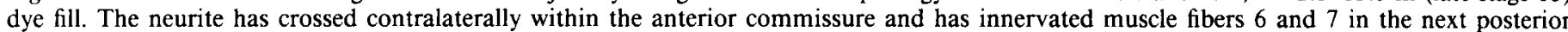

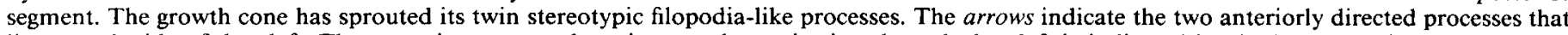

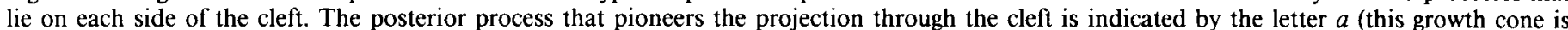

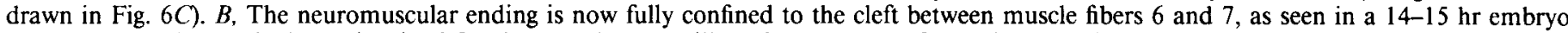

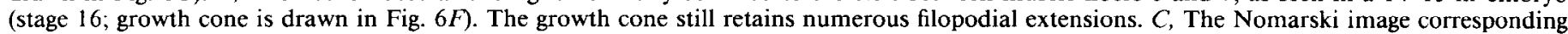
to $B$. Scale bar: $A, 7.5 \mu \mathrm{m} ; B$ and $C, 5 \mu \mathrm{m}$.

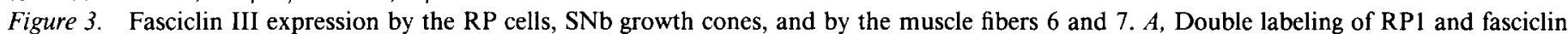

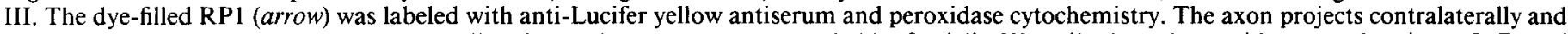

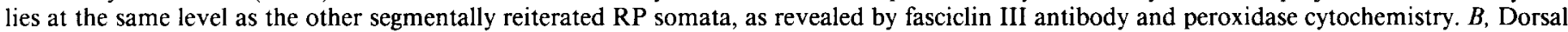

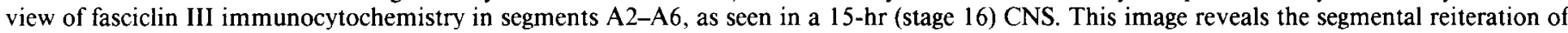

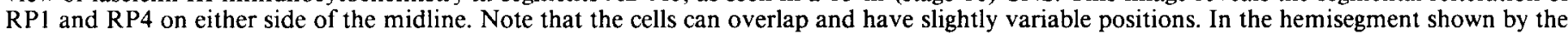

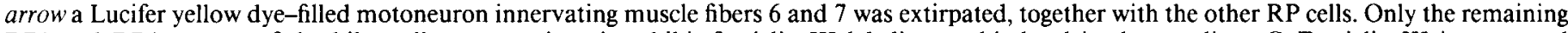

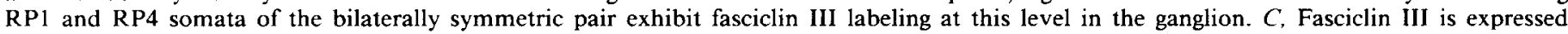

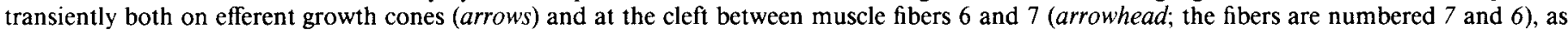

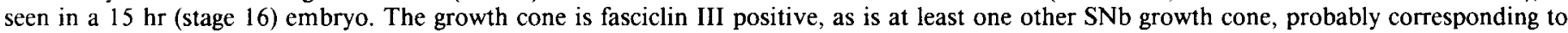

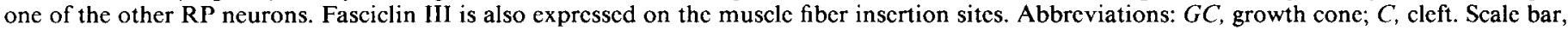
$10 \mu \mathrm{m}$ for $A$ and $B ; 16 \mu \mathrm{m}$ for $C$. 


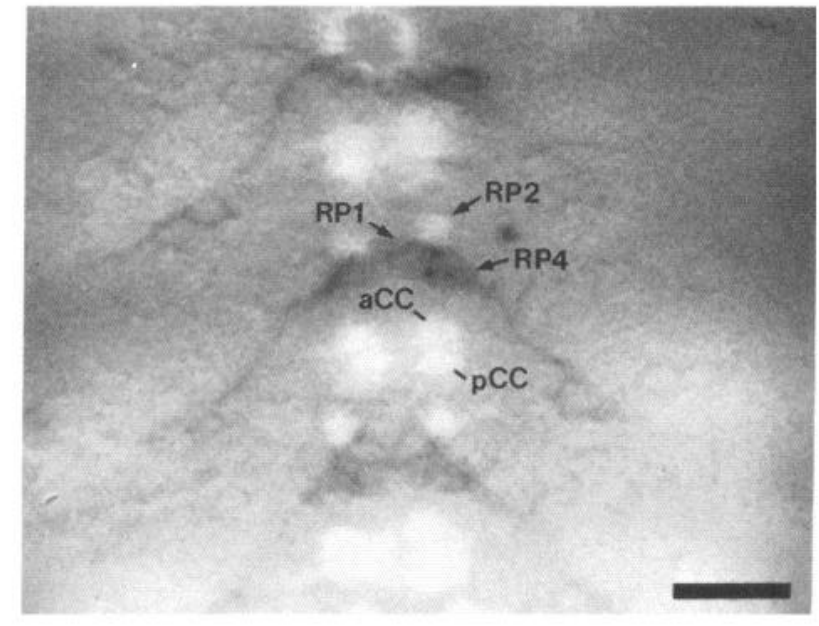

Figure 4. Characterization of the dorsal RP neurons. A double-fluorescence image showing the neurons immunopositive for even-skipped in positive image and the neurons immunopositive for fasciclin III in negative image. The composite was generated by subtracting the video image for the rhodamine fluorescing fasciclin III-positive cells from the image of the fluorescein-labeled even-skipped-immunoreactive cells. Both $R P 1$ and $R P 4$ are visible on the dorsal surface (arrows), as black fasciclin III-immunopositive cells. The even-skipped labeled RP2 (arrow) lies directly anterior to $R P I$ and $R P 4$. The paired cells $a C C$ and $p C C$ are also labeled with the even-skipped antibody. Scale bar, $10 \mu \mathrm{m}$.

$16 ; n=10$ embryos), when the growth cone contacts are made. It is no longer detected at the muscle fiber cleft by $16 \mathrm{hr}$ (stage $17 ; n=2$ embryos). Staining of the RP growth cones is also lost at this stage. The staining is not observed on any of the other muscle fibers in the abdominal hemisegment, with the exception of two ventral oblique fibers ( 15 and $16 ; n=13$ embryos), which also have a cleftlike expression pattern and have their motor endings similarly aligned on the cleft. Elsewhere in the periphery, the fasciclin III expression is observed on the surfaces of the epidermis, as first noted by Patel et al. (1987).

The muscle fiber 6 and 7 neuromuscular ending develops from site-specific filopodial contacts. We examined the events leading to synapse formation on muscle fibers 6 and 7 in a series of staged, dye-filled, and unfixed embryos. Each dye fill was used to generate a single, computer-enhanced image of the growth cone captured at a particular stage in its development. From these images $(n=22)$, we reconstructed the behavior of the motoneuron as it differentiated at its target.

During the period of 12-13 hr (stage 15), the RP3 growth cone projects peripherally between two layers of musculature, with the muscle fibers 28 and 14 on the external side and muscle fiber 7 on the internal side. The growth cone contacts the cleft of muscle fibers 6 and 7 at a level midway between the anterior and posterior segment borders. While we did not examine the dynamics of exploratory behavior by the RP3 growth cone prior to its morphogenesis at the cleft, anti-HRP immunocytochemistry (Johansen et al., 1989b; see also Fig. $1 \mathrm{~A}$ ) and vital labeling of growth cones (Chiba et al., 1990) indicate that exploring SNb growth cones can extend filopodia to lengths of up to $20 \mu \mathrm{m}$ and thus can potentially contact several muscle fibers from a single site.

The morphological changes leading to synapse formation at the cleft between muscle fibers 6 and 7 are first characterized by the generation of three prominent processes detected at 12.5$13.5 \mathrm{hr}$ (late stage 15; Figs. $2 A, 6 A-D$ ). The processes resemble filopodia in size, have predictable orientations and length (see Materials and Methods for definition), and project over specific surfaces of muscle fibers 6 and 7. Two of the stereotypic processes project anteriorly up to $5 \mu \mathrm{m}$, one apiece on muscle fibers 6 and 7 ( $n=11$ dye fills; marked by arrowheads, Fig. $6 A-D$; these processes are also indicated in the growth cone of Fig. $2 A$ by downward arrows). The two processes are thin (less than 0.5 $\mu \mathrm{m})$ and relatively straight and project in parallel on either side of the cleft separated by about $2-3 \mu \mathrm{m}$. They were not observed to cross between the two muscle fibers.

The third stereotypic process emerges from the posterior side of the growth cone, roughly midway between the sites where the anterior two processes arise. The posteriorly directed process projects along the cleft between muscle fibers 6 and 7 (the process is marked by the letter "a" in Figs. $2 A, 6 B-D$ ). It is this third process that apparently pioneers the projection of the growth cone through the cleft to the interior facing side of the muscle fibers, where the mature neuromuscular ending is found.

While each of the three stereotypic processes resembles filopodia in both caliber and length, their cytoskeletal contents remain to be characterized. We do not know how motile these processes are, although their consistent detection in growth cones at this stage of their morphogenesis suggests that they are not short-lived. In contrast to them, several filopodia not showing stereotypic orientation project both laterally and anteriorly from the growth cone. Occasionally, one or more long filopodia were observed to have crossed the anterior segmental border, apparently to contact the muscle fiber 6 and 7 homologs $(n=2$; arrow, Fig. 6A).

Within $1 \mathrm{hr}$ (early stage 16), successive dye fills reveal that the entire growth cone extends a posteriorward projection (Fig. $6 E, F$, process lettered "a") that becomes fully aligned along the cleft between muscle fibers 6 and 7 (Fig. $6 E, F ; n=4$ ). The two stereotypic anterior processes are no longer detected at this stage and presumably are retracted. Dye fills of the growth cones that have extended posteriorly down the cleft bear numerous short, laterally directed filopodial processes, together with other processes directed anteriorly (Fig. $6 E, F$ ). These filopodial processes are also transient and do not show stereotypy.

By 15-16 hr (late stage 16), dye fills show the muscle 6 and 7 ending divided into a pair of approximately equal-length neuritic processes $(n=7$; labeled " 1 " and "2," Fig. $6 G-I)$. Fills made at this time reveal few of the lateral filopodia-like processes that project from the axon proximal to the branch point, resulting in a smooth appearance. By $17 \mathrm{hr}$ of development (early stage 17), the neuromuscular ending occupies its mature extent along the two target muscle fibers (Fig. $6 G-I$ ). Fine processes often cross between the two branches $(n=6)$, a feature also found in mature larval endings. Late-stage dye fills display axonal swellings that resemble synaptic boutons (Figs. $6 H, I$; $7 A$ ). At this time, immature endings label with antisera to the neurotransmitter glutamate (Johansen et al., 1989b), and the muscle fibers contract in response to CNS activity (Anderson and Keshishian, 1990).

By $17 \mathrm{hr}$ (stage 17) of embryogenesis (Fig. $7 A$ ), the overall motoneuronal ending anatomy resembles that seen in a third instar larva (Fig. 7B). The motoneuron divides its ending equally between the two muscle fibers and furthermore is mirror symmetrical over their surfaces, with the line of reflection being the cleft. Thus, the symmetry extends both to the early behavior of growth cone filopodia as well as to the later posteriorward bifurcation of the ending. 
Johansen et al. (1989a,b) found that in the anterior abdominal segments of both embryos and larvae the neuromuscular endings on muscle fibers 6 and 7 extend equally in both anterior and posterior directions from the nerve entry point. As the embryonic growth cone and synaptic contact project largely in a posterior direction over muscle fibers 6 and 7 , we predict that in anterior segments muscle fibers 6 and 7 are coinnervated with at least one other motoneuron, or that novel anterior branches are generated over the muscle fibers in later development.

In conclusion, RP3's growth cone establishes a motor ending on muscle fibers 6 and 7 over a period of about 2 hr. During this period, the growth cone resides exclusively on and about the cleft region of the muscle fibers and develops into a branched structure that prefigures the mature larval ending. This is accomplished by a series of predictable shape changes, including the genesis of stereotypic filopodial processes and the growth of processes in specific directions.

Synaptic morphogenesis on muscle fiber 13. To what extent are the features of synapse formation seen at muscle fibers 6 and 7 shared by other motoneurons? We examined in detail the innervation of muscle fiber 13 , which lies immediately adjacent to muscle fiber 6 (Figs. $1 B, 5$ ). The peripheral axonal growth and innervation of muscle fiber 13 occurs at approximately the same time and proceeds at the same rate as the events described above for muscle fibers 6 and 7 .

As the trajectory in the periphery to muscle fiber 13 extends over the relative wide expanse of muscle fiber 6 , we reconstructed how the RP1 growth cones behave over nontarget muscle fiber surfaces. Figure $8 A-D$ illustrates four dye-filled RP 1 growth cones located on the externally facing side of muscle fiber 6 prior to contacting the muscle fibcr $13 \mathrm{mcmbranc}$ surface. Growth cones over this part of the trajectory were not characterized by the presence of stereotypic processes. In addition to variably directed filopodia, the size of the growth cone lamellipodia varied considerably between preparations $(n=6$; compare Fig. $8 A, B$ to $C, D)$. Here the growth cones are positioned on the side of muscle fiber 6 facing the embryo's cuticle, opposite to the side where RPI makes its ending. This location agrees with observations made of whole nerve trajectories in embryos by Johansen et al. (1989b), where growth cone filopodia were seen to spread from the efferent $\mathrm{SNb}$ nerve along the externally facing surfaces of muscle fiber 6 .

In contrast to their morphology on the muscle fiber 6 surface, RPl growth cone dyefills show a consistent change in appearance as they approach the edge of fiber 13. Just as RP3 growth cones sprout stereotypic filopodia-like projections when they reach the musclc fiber 6 and 7 cleft, RP1 growth cones align along the muscle fiber 13 edge and project a single long, posteriorly directed process ( $n=8$; Fig. $8 E-H$. labeled "a"). A similar morphological response was seen in RP4 growth cones at this point $(n=4)$. This "exploratory" process is generated while the body of the RP3 growth cone is still on the external facing side of muscle fiber 6 . The process extends along the side of muscle fiber 13 facing the embryonic interior, where the mature synaptic contacts are located. Thus, like the behavior at the muscle fiber 6 and 7 cleft, muscle fiber 13 growth cones project to the interior-facing side when they reach their targets. The posteriorward filopodial growth will subsequently mature into the principal projection of RP1 on muscle fiber 13. Other variable filopodia are observed during the transitional phase. These filopodia are subsequently lost as the contact further differentiates.

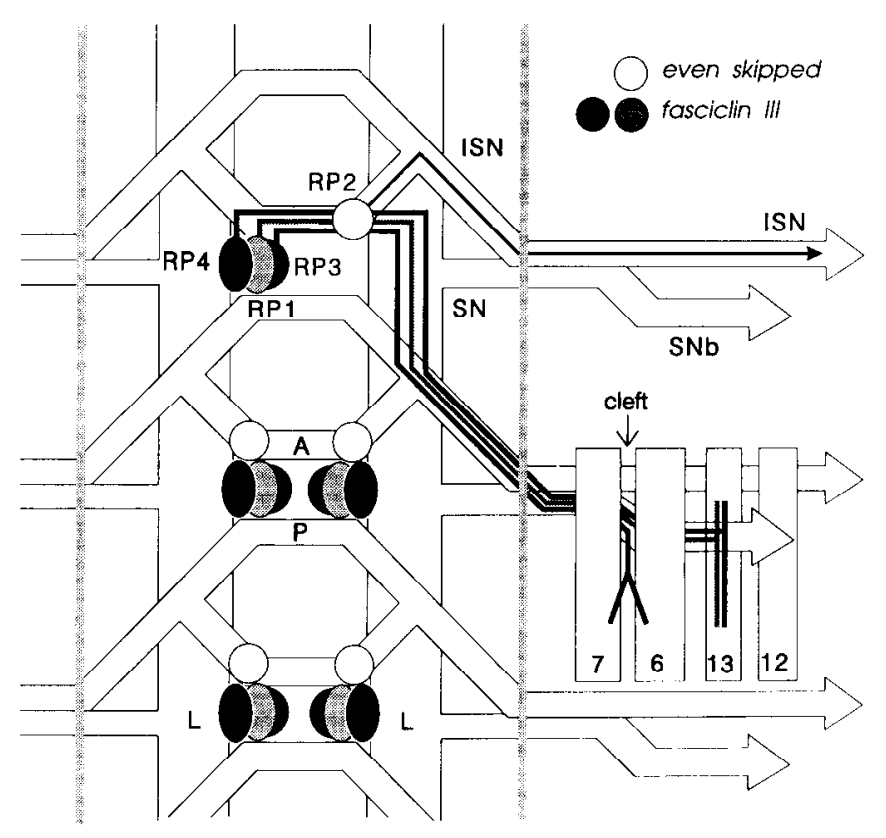

Figure 5. A schematic representation of the RP trajectories. RPI and $R P 3$ share similar central projections, crossing anteriorly and contralaterally to pioncer the anterior commissure (Jacobs and Goodman, 1989). At the longitudinal connective, the growth cones turn posteriorly and follow the $I S N$ out of the neuropil (Thomas et al., 1984; Patel et al., 1987). Both axons exit the CNS and innervate their respective muscle fibers by the $S N b$ branch. $R P 4$ resembles $R P l$ in both its central trajectory and choice for peripheral target. By contrast, $R P 2$ projects ipsilaterally to contact the $I S N$ tract and ultimately innervates dorsal muscle fiber targets of its own segment ( $n=4$ dye fills). Vertical gray bars mark the border of the CNS. The trajectories and endings correspond to a stage 17 embryo, as viewed in a fillet, from the inside of the embryo. Abbrev.: $A, P$, anterior, posterior commissure; $L$, longitudinal connective.

The motoneuron's branching over the target muscle fiber follows a predictable sequence $(n=7$; Figs. $8 I-L, 9 A)$. The neuromuscular ending has up to two anterior-lateral branches in addition to its posteriorward projection. The anterior-lateral branches arise after the posteriorward projection has extended some $5-10 \mu \mathrm{m}$. Bouton-like varicosities are observed by $16 \mathrm{hr}$ (stage 17; Figs. 8L, 9B).

Thus, over a 1-2 hr period during late stage 16 , the motoneuron's growth cone follows a precise, orchestrated transition into a differentiated motor ending. A comparison of the embryonic synapse with the mature neuromuscular ending in a third instar larva is shown in Figure $9 B, C$. Mature muscle fiber 13 endings (Johansen et al., 1989a) usually have multiple posteriorward branches lying adjacent and parallel to each other. It is probable that these projections in larvae represent in part the endings of RP1 and RP4.

\section{Discussion}

In this article, we have used computer-enhanced digital optical microscopy, dye fills, and immunocytochemistry to deduce the growth cone and filopodial behavior of identified Drosophila motoneurons. The differentiation and fascicle choices of the RP neurons within the CNS have been examined previously, and the cells were described as efferents, although their targets were not determined (Thomas et al., 1984; Jacobs and Goodman, 1989). Recently Sink and Whitington (1991) have identified the targets of numerous abdominal motoneurons. 

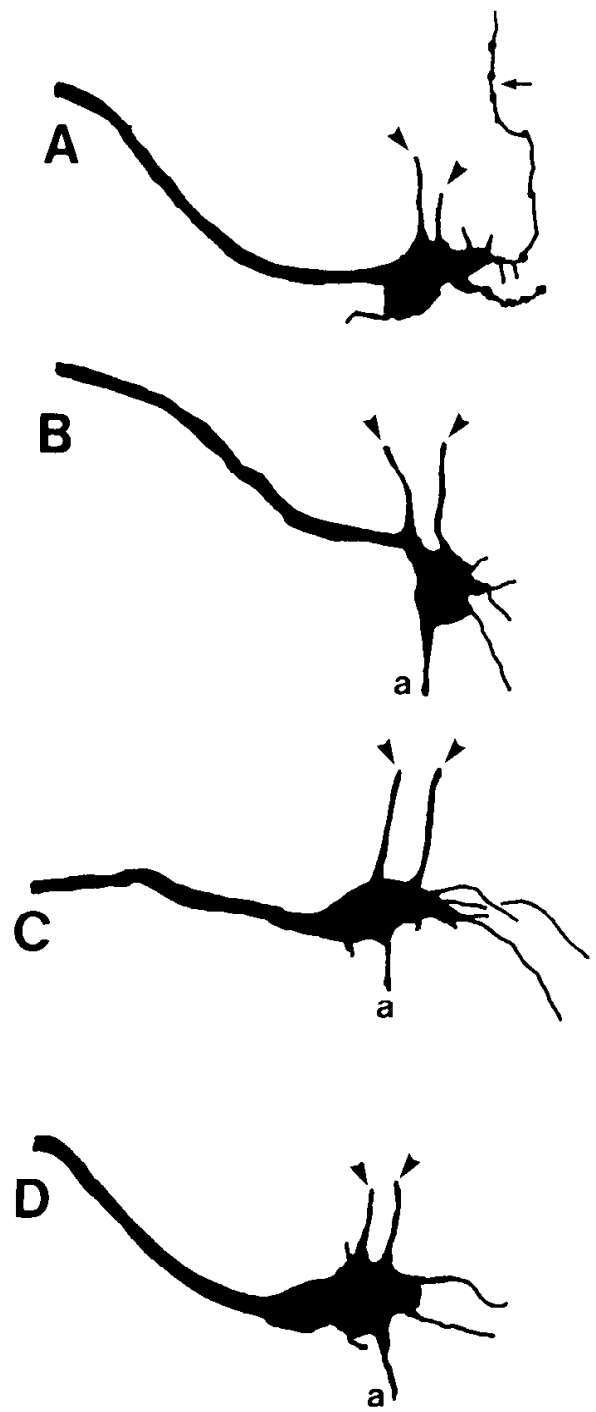
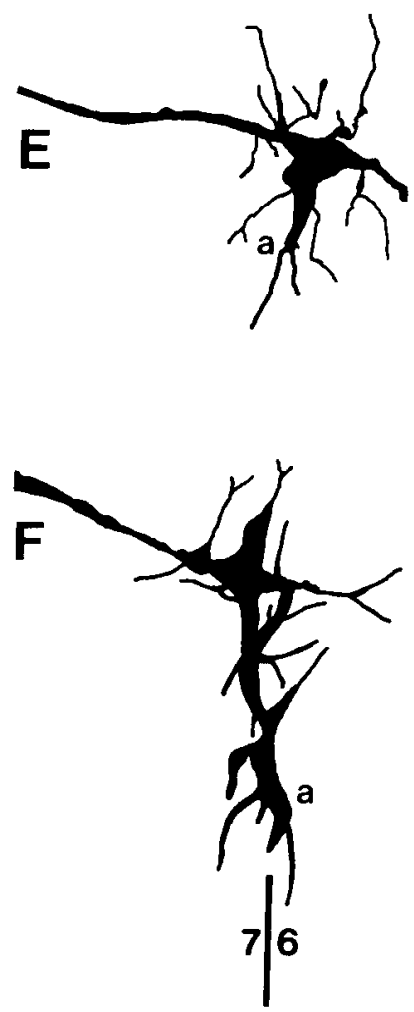
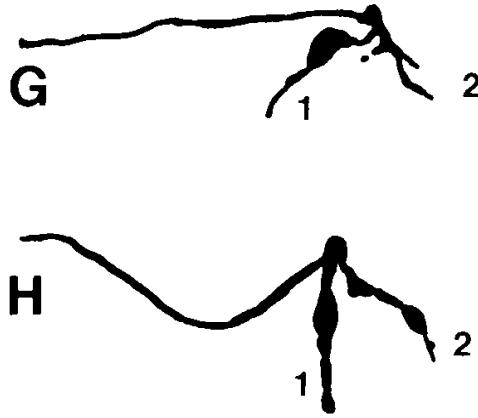

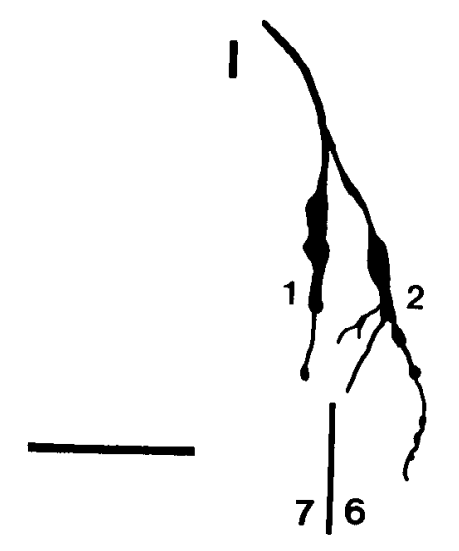

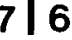

Figure 6. Growth cone behavior leading to synapse formation by RP3 on muscle fibers 6 and 7 . These tracings were made from enhanced video images of dye-filled growth cones from a series of unfixed embryos. The drawings in this figure are positioned in three columns, within which they are comparably aligned. The vertical bar under each column denotes the border between muscle fibers 6 and 7 (the cleft). $A-D$, Stereotypic filopodial projections of the growth cone seen as it first contacts muscle fibers 6 and 7 , during 12.5-13.5 hr (late stage 15). Two prominent, stereotypic filopodia (arrowheads in $A-D$ ) are evident in these growth cones. At this time, some filopodia (arrow, $A$ ) can cross the anterior segment border. However, the small posteriorward process (labeled $a, B-D$ ) is fated to pioneer the principal posteriorward growth of the neuronal ending. The other filopodia seen are variable in orientation and number. $E$ and $F$, The posteriorward elongation of the growth cone along the muscle fiber 6 and 7 cleft. In 14-15 hr (stage 16) embryos, the growth cone advances posteriorly $(a)$ to extend up to $10 \mu \mathrm{m}$ from its initial entry point. During this stage, the ending bears numerous fine and variably dirceted filopodia. $G-I$, The subdivision of the motoncuronal cnding into symmetrical muscle fiber 6 and 7 projections. At 15-16 hr (late stage 16), the ending divides into two equally long branches, running posteriorly along each muscle fiber labeled $l$ and 2 ). At this stage, there is substantial trimming of finer lateral filopodia, especially those emerging from the axon behind the branch point. This stage is also associated with the elaboration of synaptic boutons. Scale bar, $10 \mu \mathrm{m}$.

Connectivity is precise. The Drosophila motoneurons we examined innervate specific muscle fibers with high reliability. We did not see random collateral axon branches to other muscle fibers that are later withdrawn, although filopodia can contact multiple muscle fibers. In support of the reliability of motoneuronal targeting is the extensive anatomical stereotypy of neuromuscular endings seen using neuron-specific antisera (Johansen et al., 1989a). In addition, Chiba et al. (1990) showed that there is a stereotypic pattern in the CNS of backfilled cell bodies when muscle fibers are fluorescently labeled with the lipophilic dye Dil. Similarly, Sink and Whitington (1991) have observed, in addition to their dye fills, consistent populations of cell bodies when specific nerve trunks are backfilled with Dil or cobalt.

One consequence of precision in connectivity is that the CNS can independently control individual muscle fibers. Also, because Drosophila motoneurons can express one or more of sev- 

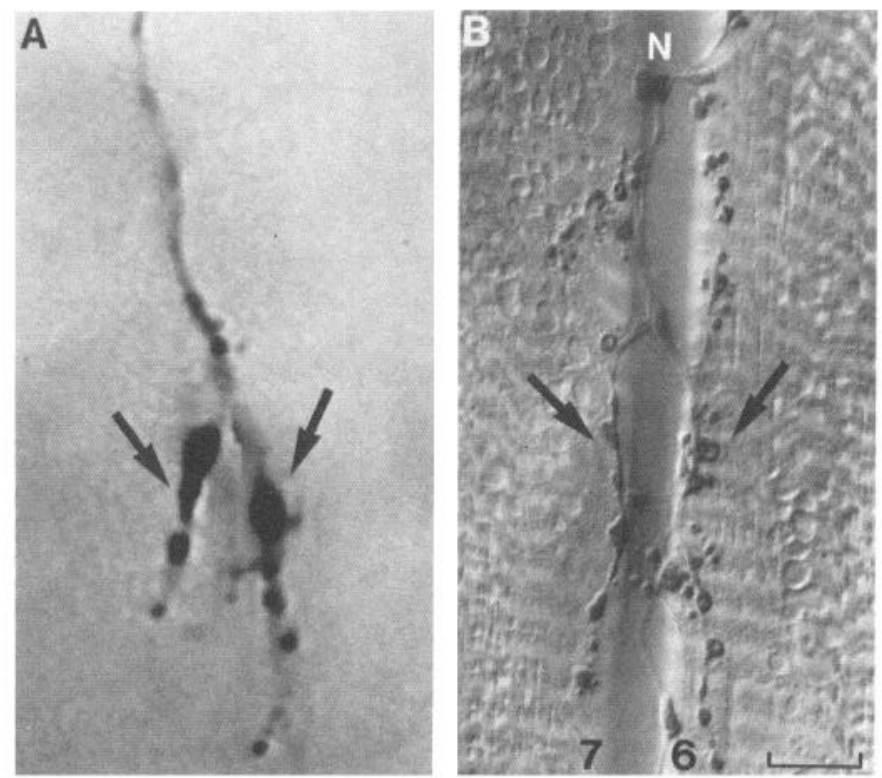

Figure 7. A comparison of the embryonic and mature innervation anatomy on muscle fibers 6 and 7. $A$, In a dye fill at 16-17 hr (stage 17), the principal features of the mature postembryonic neuromuscular anatomy are evident, including the bifurcation of the axon (arrows) to the two targets, the finer processes crossing between the muscle fibers, and the distribution of synaptic boutons (this video image of the motoneuron's arborization corresponds to the tracing in Fig. $6 I$ ). $B$, In a third instar larva, the characteristic neuromuscular arborization on muscle fibers 6 and 7 , corresponding to the region innervated in the embryo (arrows), is labeled with a glutamate antiserum and peroxidase cytochemistry (Johansen et al., 1989a). The letter $N$ corresponds to the RP1 branch point from the SNb. Scale bar: $5 \mu \mathrm{m}$ for $A ; 20 \mu \mathrm{m}$ for $B$.

eral transmitters, including glutamate (Johansen et al., 1989a), proctolin (Anderson et al., 1988), and octopamine (Halpern et al., 1988), muscle fibers may be specifically excited and modulated in complex ways.

A key to understanding connectivity in the Drosophila neuromuscular system is the analysis of the specialized properties of the individual muscle fiber membranes. In Drosophila, embryonic motoneurons not only grow directly to the correct muscle fibers but also project to favored sites on their target cell surfaces. These sites are apparently ignored by other motoneurons, which must follow the same nerve pathways en route to their own muscle fiber targets. Only one of these contacts will result in the formation of a synapse. Through unknown mechanisms, each growth cone leaves the nerve to innervate a preferred muscle fiber. This suggests that motoneurons recognize cell-specific surface features as they contact different muscle fibers.

Is there evidence for the specialization of Drosophila muscle fibers at the molecular level? There are two examples of $\mathrm{Dro}$ sophila muscle fiber subsets expressing specific molecules during the period of embryonic synaptogenesis. Harkins et al. (1990) have identified genes using enhancer detector methods, where the protein expression is restricted to specific embryonic Drosophila muscle fibers. The specialized expression is limited, for some genes, to a single muscle fiber. In this article, we described fasciclin III expression by two pairs of muscle fibers, the ventral longitudinals 6 and 7 and the ventral obliques 15 and 16 , in a temporally and spatially restricted fashion just prior to and during synaptogenesis. For both muscle fiber pairs, the fasciclin III expression is found on membrane surfaces where the synapses will be made. Furthermore, the expression is also seen on efferent growth cones, including in one case the presynaptic motoneuron.

Fasciclin III has been shown to mediate a strong homophilic adhesion between tissue culture cells induced to express the molecule on their surfaces (Snow et al., 1989). As the protein is expressed in some cases on both the growth cone and target membrane surface region during synaptogenesis, it is possible that homophilic adhesion mechanisms may also be occurring in the embryo. However, a simple mechanism to explain RP1 connectivity based solely on the homophilic adhesion of fasciclin III is insufficient, as the protein is also expressed at the muscle fiber apodemes, and because at least one other $\mathrm{SNb}$ neuron not targeted to muscle fibers 6 and 7 also expresses fasciclin III on its growth cone. The phenotype of fasciclin III mutants at neuromuscular junctions has not been reported. The null mutant is not lethal (Snow et al., 1989), although as is seen for fasciclin I (Elkins et al., 1990), the severity of the fasciclin III null phenotype could depend on the activity of other loci.

The dynamic expression of cell-surface molecules on specific regions of developing neurons appears to be universal (Jessel, 1988). Some of the proteins involved are expressed regionally on neural surfaces at times appropriate for a role in pathfinding and are often attractive or adhesive in function (Bastiani et al., 1987; Patel et al., 1987; Dodd et al., 1988; Snow et al., 1989; Furley et al., 1990; Jay and Keshishian, 1990). Numerous proteins thought to mediate cell adhesion have sequence homologs in Drosophila or grasshopper, including laminin (Montell and Goodman, 1988; Garzino et al., 1989), fibronectin (Gratecos et al., 1988), integrins (Leptin et al., 1989), and members of the immunoglobulin superfamily (Harrelson and Goodman, 1988; Seeger et al., 1988; Bieber et al., 1989). A role for these proteins in Drosophila neuromuscular development remains to be determined.

Our observation that Drosophila muscle fibers possess molecularly heterogeneous cell surfaces is consistent with the cell biology of muscle fibers. Cell adhesion molecules, such as NCAM and cadherin, are dynamically expressed at developing vertebrate neuromuscular junctions in the course of synaptogenesis (Rieger et al., 1985; Bixby et al., 1987). Specific proteins are also known to be preferentially associated with muscle basal lamina at sites of neuromuscular junctions, although they may be secreted there by other cells (Sanes, 1989). S-laminin is concentrated in synaptic clefts at muscle fibers and has been shown to promote motoneuronal adhesion in in vitro assays (Hunter et al., 1989). Finally, in syncytial muscle cells, specific transmembrane proteins are localized to the vicinity of the nuclei from which they were derived (Hall and Ralston, 1989; Pavlath et al., 1989). A similar strategy could be used in Drosophila muscle fibers.

Growth cones generate stereotypic filopodia when they make trajectory changes. In this study, we reconstructed the behavior of two motoneurons as they encountered their targets through a comparison of a succession of dye fills in staged embryos. An outstanding feature of both RP1 and RP3 is that their growth cones make characteristic shape changes, turn in specific directions, and sprout fine, stereotypic processes as they grow on their respective targets.

Filopodial preferences for specific axon surfaces have been described by Raper et al. (1983) for central neurons of grasshopper embryos. Similarly, the Til pioneer neurons of grass- 

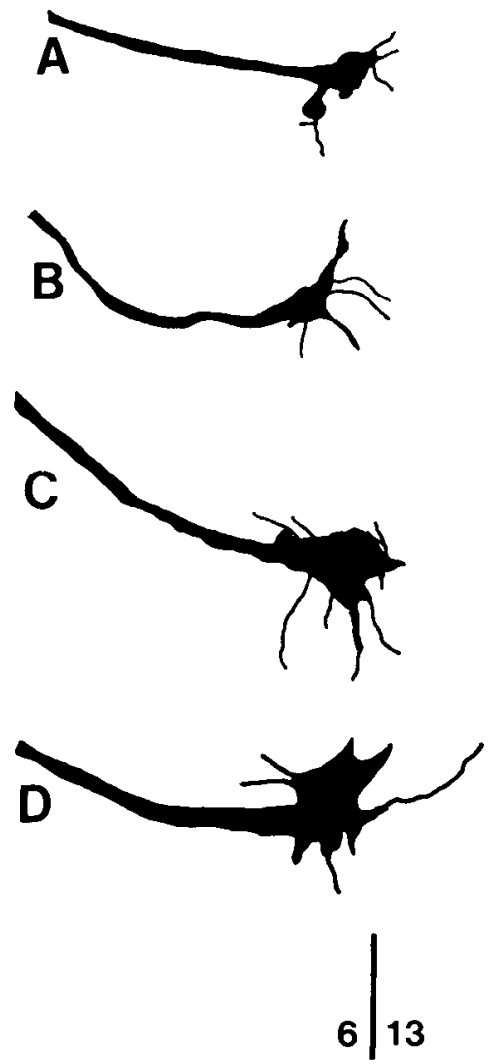

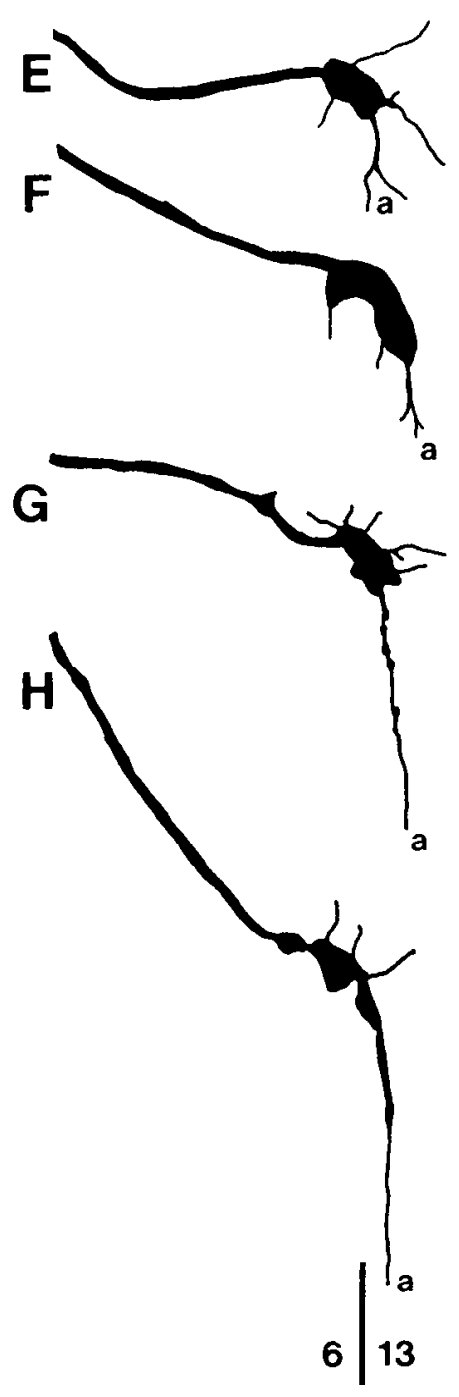

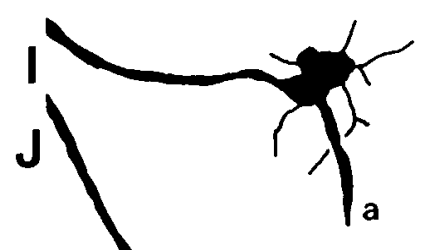
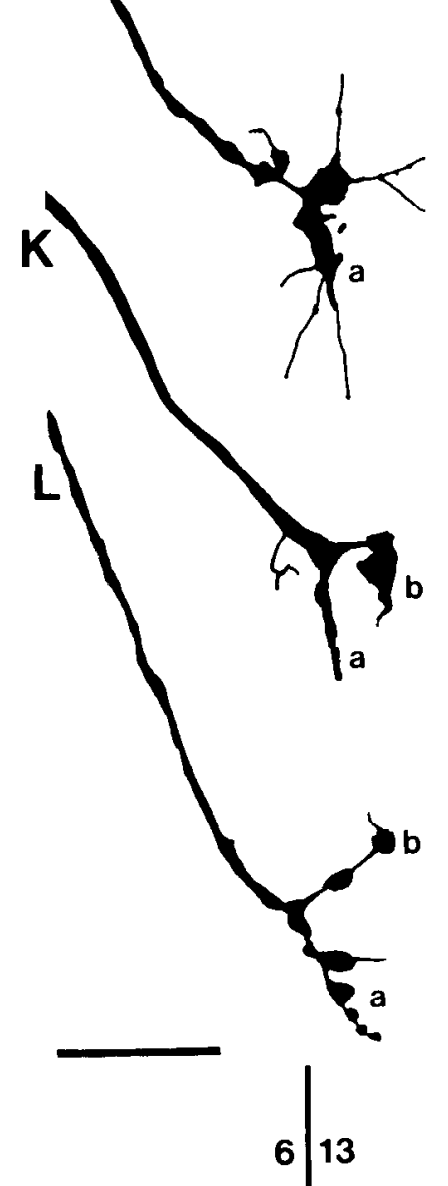

Figure 8. Growth cone behavior leading to synapse formation by RP1 on muscle fiber 13. These tracings were made from enhanced video images of dye-filled growth cones from a series of unfixed embryos. The drawings in this figure are aligned as in Figure 6 . The vertical bar under each column denotes the border between muscle fibers 6 and 13. $A-D$, The RPl growth cone prior to contact with muscle fiber 13 . In each of these examples, the growth cone was positioned on the externally facing surface of muscle fiber 6 and has not yet reached the edge of muscle fiber 13 , the axonal target. $E-H, \mathrm{RPl}$ growth cones make a stereotypic posteriorward projection when they first contact the edge of muscle fiber 13 . The projection (labeled $a$ ) is led by a single filopodium on the side of muscle fiber 13 facing the embryo's interior, while the growth cone body is still on the cuticle-facing side of muscle fiber 6 . I-L, Later differentiation of the ending on muscle fiber 13. Anterior-lateral projections arise (labeled $b$ ) after the elongation of the posteriorward projection (labeled $a$ ). Scale bar, $10 \mu \mathrm{m}$.

hopper limb buds show filopodial projections with characteristic surface preferences during their proximal growth (Bentley and Caudy, 1983; Caudy and Bentley, 1986; O'Connor et al., 1990). In both of these examples, the direction or surface selected by a growth cone is predicted by the behavior of its filopodia. Thus, in the CNS, specific axonal fasciculations are anticipated by filopodial preferences, while for the limb bud pioneer neurons the growth cones respond to filopodial contacts made on specific guideposts or on segmental borders. O'Connor et al. (1990) have shown that single filopodial contacts on a guidepost neuron can redirect the trajectory of a growth cone. A similar redirection of the RP axons occurs as they first contact their appropriate targets.

In Drosophila, we have found that the behavior of the RP1 and RP3 growth cones at their respective targets is predictable.
Stereotypic projections are associated with important trajectory changes. For example, a critical step in the innervation of muscle fibers 6 and 7 is the turn made posteriorly at the cleft between the two fibers. In growth cones we have dye filled at this stage, two anteriorly directed processes are seen, arrayed one apiece on each of the two fibers. The posteriorward projection is initiated by a third process projecting opposite to the anterior two. The fact that the growth cone is arrayed in this manner when it turns $90^{\circ}$ posteriorly suggests that these projections are part of the necessary movements needed to shift the motoneuron from the $\mathrm{SNb}$ nerve tract to innervate a specific target. RPI also shows this kind of behavior when it initiates its posteriorward turn on muscle fiber 13 , with a single prominent process running down the medial edge of the target.

The branching of motoneuronal endings during the latter stages 

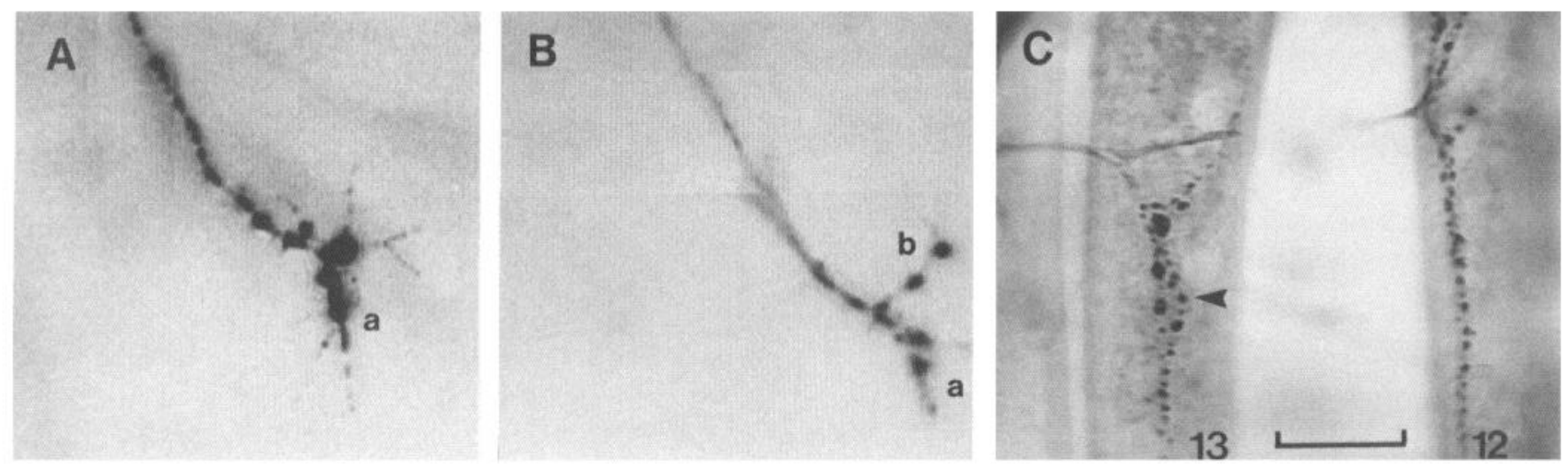

Figure 9. Video-enhanced images of growth cone dye fills on muscle fiber 13. A, In a 16-17 hr (late stage 16) embryo, fine filopodial projections are directed posteriorly from an RP1 growth cone (drawn in Fig. 8J). One of these is initiating the posteriorward projection $(a)$. Dye fills of RP4 growth cones at this stage have a similar morphology. $B$, View of RP1's growth cone at stage 17, showing the formation of the anterior projection $(b)$, in addition to the initial posteriorward projection $a$ (drawn in Fig. $8 L$ ). Synaptic boutons are also present. $C$, For comparison, neuromuscular endings on muscle fibers 13 (arrowhead) and 12 in a third instar larva are shown labeled with glutamate antiserum and peroxidase cytochemistry. Scale bar: $5 \mu \mathrm{m}$ for $A ; 8 \mu \mathrm{m}$ for $B ; 50 \mu \mathrm{m}$ for $C$

of synaptogenesis is also precise. For both RP1 and RP3 both the location and the sequence of branching are predictable. Thus, at muscle fibers 6 and 7 the bifurcation of the ending is made after the posteriorward process has advanced some $5 \mu \mathrm{m}$ down the cleft. In the case of RP1, its anteriorward branches are generated after the primary posteriorward process has been established. Also, the anterior branch is generated from the site where the growth cone first contacts the muscle fiber. How branch generation is controlled in this fashion is not known.

In all likelihood, the predictable behavior of growth cone processes arises in response to features of the muscle fiber surfaces. Reshaping of growth cones by slight differences in filopodial adhesion has been well described and is believed to be linked to the reorganization of cytoskeletal components (Letourneau, 1985; Mason, 1985; Bray, 1987; Smith, 1988). Preferential filopodial adhesion could also precede the genesis of stereotypic processes, the branching of Drosophila motoneurons over a single target membrane, and the splitting of a single growth cone, as in the situation at muscle fibers 6 and 7 , to equally adhesive surfaces on its two target cells. The symmetrical distribution of fasciclin III on both muscle fibers is consistent with this mechanism.

However, how extracellular signals regulate the motile behavior of growth cones is less well understood. There is evidence that neurotransmitters can modulate growth cone morphology and motility (Kater et al., 1988). The role of synaptic activity in the differentiation of Drosophila neuromuscular connections is an open question. Nevertheless, as with other developing efferent growth cones (Sun and Poo, 1987; Haydon and Zoran, 1989), we do know that neurotransmitter is concentrated in Drosophila embryonic growth cones (Johansen et al., 1989b) and that the muscle fibers contract in response to CNS activity during the later stages of growth cone differentiation (Anderson and Keshishian, 1990). It has been shown genetically that activity may be an important factor in the shaping of motor endings observed in Drosophila larvae, influencing both the length of processes and the number and placement of synaptic boutons (Budnik et al., 1989, 1990).

In conclusion, we have examined the cellular events governing the development of synaptic connections in Drosophila embryos. Motoneuron growth cones exhibit precise targeting and pro- ject to specific muscle fibers through active growth cone exploration. Growth cones are capable of generating precise and stereotypic processes that are associated with trajectory changes on the target surfaces. Finally, we have provided evidence that Drosophila muscle fibers possess molecularly heterogeneous cell surfaces that potentially are involved in growth cone recognition of appropriate targets.

\section{References}

Anderson H (1988) Drosophila adhesion molecules and neural development. Trends Neurosci 11:472-475.

Anderson MS, Keshishian H (1990) The role of postsynaptic activity in establishing appropriate synaptic morphology during neuromuscular development in Drosophila embryos. Soc Neurosci Abstr 16: 973.

Anderson MS, Halpern ME, Keshishian HS (1988) Identification of the neuropeptide transmitter proctolin in Drosophila larvae. Characterization of muscle fiber-specific neuromuscular endings. J Neurosci 8:242-255.

Bastiani MJ, Harrelson AL, Snow PM, Goodman CS (1987) Expression of fasciclin I and II glycoproteins on subsets of axon pathways during neuronal development in the grasshopper. Cell 48:745-755.

Bentley D, Caudy M (1983) Navigational substrates for peripheral pioneer growth cones: limb-axis polarity cues, limb-segment boundaries and guidepost neurons. Cold Spring Harbor Symp Quant Biol 48:573-585.

Bentley D, Keshishian H (1982) Pathfinding by peripheral pioneer neurons in the grasshopper. Science 218:1082-1088.

Bieber AJ, Snow PM, Hortsch M, Patel NJ, Jacobs JR, Traquina ZR, Schilling J, Goodman CS (1989) Drosophila neuroglian: a member of the immunoglobulin superfamily with extensive homology to the vertebrate neural adhesion molecule L1. Cell 59:447-460.

Bixby JL, Pratt RS, Lillien J, Reichardt LF (1987) Neurite outgrowth on muscle cell surfaces involves extracellular matrix receptors as well as $\mathrm{Ca}^{2+}$-dependent and -independent cell adhesion molecules. Proc Natl Acad Sci USA 84:2555-2559.

Bray D (1987) Growth cones: do they pull or are they pushed? Trends Neurosci 10:431-433.

Budnik V, Wu C-F, White K (1989) Altered branching of serotonincontaining neurons in Drosophila mutants unable to synthesize serotonin and dopamine. J Neurosci 9:2866-2877.

Budnik V, Zhong Y, Wu C-F (1990) Morphological plasticity of motor axons in Drosophila mutants with altered excitability. J Neurosci 10: 3754-3768.

Campos-Ortega JA, Hartenstein V (1985) The embryonic development of Drosophila melanogaster. Berlin: Springer.

Canal I, Ferrús A (1986) The pattern of early neuronal differentiation in Drosophila melanogaster. J Neurogenet 3:293-319. 
Caudy M, Bentley D (1986) Pioneer growth cone steering along a series of neuronal and non-neuronal cues of different affinities. J Neurosci 6:1781-1795.

Chiba A, Chang T, Halpern ME, Keshishian H (1990) Growth cones in Drosophila show stereotypic morphology and behavior. Soc Neurosci Abstr 16:625.

Crossley CA (1978) The morphology and development of the Drosophila muscular system. In: The genetics and biology of Drosophila, Vol 2b (Ashburner M, Wright TRF, eds), pp 499-560. New York: Academic.

Dodd J, Morton SB, Karagogeos D, Yamamoto M, Jessell TM (1988) Spatial regulation of axonal glycoprotein expression on subsets of embryonic spinal neurons. Neuron 1:105-116.

Doe CQ, Smouse D, Goodman CS (1988) Control of neuronal fate by the Drosophila segmentation gene even-skipped. Nature 333:376378.

Eisen JS, Myers PZ, Westerfield M (1986) Pathway selection by growth cones of identified motoneurons in live zebrafish. Nature 320:269_ 271.

Elkins T, Zinn K, McAllister L, Hoffmann FM, Goodman CS (1990) Genctic analysis of a Drosophila ncural cell adhesion molccule: interaction of fasciclin I and Abelson tyrosine kinase mutations. Cell $60: 565-575$

Frasch M, Hoey T, Rushow C, Doyle H, Levine M (1987) Characterization and localization of the even-skipped protein of Drosophila. EMBO J 6:749-759.

Fujita SC, Zipursky SL, Benzer S, Ferrús A, Shotwell SL (1982) Monoclonal antibodies against the Drosophila nervous system. Proc Natl Acad Sci USA 79:7929-7933

Furley AJ, Morton SB, Manalo D, Karagogeos D, Dodd J, Jessell TM (1990) The axonal glycoprotein TAG-1 is an immunoglobulin superfamily member with neurite outgrowth-promoting activity. Cell 61:157-170.

Garzino V, Berenger H, Pradel J (1989) Expression of laminin and of a laminin-related antigen during early development of Drosophila melanogaster. Development 106:17-27.

Giloh H, Sedat JW (1982) Fluorescence microscopy: reduced photobleaching of rhodamine and fluorescein protein conjugates by $n$-propyl gallate. Science 217:1252-1255.

Goodman CS, Bastiani MJ, Doe CQ, du Lac S, Helfand SL, Kuwada JY, Thomas JB (1984) Cell recognition during neuronal development. Science 225:1271-1279.

Gratecos D, Naidet C, Astier M, Thiery JP, Sémériva M (1988) Drosophila fibronectin: a protein that shares properties similar to those of its mammalian homologue. EMBO J 7:215-223.

Hall ZW, Ralston E (1989) Nuclear domains in muscle cells. Cell 59: $771-772$.

Halpern ME, Keshishian H (1990) Development of site-specific synapses by the Drosophila motoncuron RP1 involves stcrcotypic filopodial contacts during embryogenesis. Soc Neurosci Abstr 16:625.

Halpern ME, Anderson MS, Johansen J, Keshishian H (1988) Octopamine immunoreactive nerve terminals are found on a single identified muscle fiber of the Drosophila larval bodywall. Soc Neurosci Abstr 14:383.

Harkins E, Fluet A, Farrell E, Keshishian H (1990) Enhancer trap mutagenesis of the embryonic neuromuscular system in Drosophila melanogaster. Soc Neurosci Abstr 16:303.

Harrelson AL, Goodman CS (1988) Growth cone guidance in insects: fasciclin II is a member of the immunoglobulin superfamily. Science 242:700-708.

Haydon PG, Zoran MJ (1989) Formation and modulation of chemical connections: evoked acetylcholine release from growth cones and neurites of specific identified neurons. Neuron 2:1483-1490.

Hertweck H (1931) Anatomie und Variabilität des Nervensystems und der Sinnesorgane von Drosophila melanogaster (Miegen). Z Wiss Zool 139:559-663.

Hunter DD, Shah V, Merlie JP, Sanes JR (1989) A laminin-like ad- hesive protein concentrated in the synaptic cleft of the neuromuscular junction. Nature 338:229-234.

Jacobs JR, Goodman CS (1989) Embryonic development of axon pathways in the Drosophila CNS. I. Behavior of pioneer growth cones. J Neurosci 9:241 2-2422.

Jan LY, Jan YN (1976) Properties of the larval neuromuscular junction in Drosophila melanogaster. J Physiol (Lond) 262:189-214.

Jay DG, Keshishian HS (1990) Laser inactivation of fasciclin I disrupts cell adhesion of grasshopper pioneer neurons. Nature 348:548-550.

Jessel TM (1988) Adhesion molecules and the hierarchy of neuronal development. Neuron 1:1-13.

Johansen J, Halpern ME, Johansen KM, Keshishian H (1989a) Stereotypic morphology of glutamatergic synapses on identified muscle cells of Drosophila larvae. J Neurosci 9:710-725.

Johansen J, Halpern ME, Keshishian H (1989b) Axonal guidance and the development of muscle fiber-specific innervation in Drosophila embryos. J Neurosci 9:4318-4332.

Kater S, Mattson MP, Cohan C, Connor J (1988) Calcium regulation of the neuronal growth cone. Trends Neurosci 11:315-321.

Kuwada J (1986) Cell recognition by neuronal growth cones in a simple vertebrate embryo. Science 233:740-746.

Landmesser LT (1980) The generation of neuromuscular specificity. Annu Rev Neurosci 3:279-302.

Leptin M, Bogaert T, Lehmann R, Wilcox M (1989) The function of PS integrins during Drosophila embryogenesis. Cell 56:401-408.

Letourneau PC (1985) Axonal growth and guidance. In: Molecular bases of neural development (Edelman GM, Gall WE, Cowan WM, eds), pp 269-293. New York: Wiley.

Mason C (1985) How do growth cones grow? Trends Neurosci 8:304306

Montell DJ, Goodman CS (1988) Drosophila substrate adhesion molecule: sequence of laminin B1 chain reveals domains of homology with mouse. Cell 53:463-473.

O'Connor TP, Duerr JS, Bentley DR (1990) Pioneer growth cone steering decisions mediated by single filopodial contacts in situ. J Neurosci 10:3935-3946.

Patel NH, Snow PM, Goodman CS (1987) Characterization and cloning of fasciclin III: a glycoprotein expressed on a subset of neurons and axon pathways in Drosophila. Cell 48:975-988.

Pavlath GK, Rich K, Webster SG, Blau HM (1989) Localization of muscle gene products in nuclear domains. Nature 337:570-573.

Raper JA, Bastiani M, Goodman CS (1983) Pathfinding by neuronal growth cones in grasshopper embryos. II. Selective fasciculation onto specific axonal pathways. J Neurosci 3:31-41.

Rieger F, Grumet M, Edelman GM (1985) N-CAM at the vertebrate neuromuscular junction. J Cell Biol 101:285-293.

Sanes JR (1989) Extracellular matrix molecules that influence neural development. Annu Rev Neurosci 12:491-516.

Seeger MA, Haffley L, Kaufman TC (1988) Characterization of amalgam: a member of the immunoglobulin superfamily from Drosophila. Cell 55:589-600.

Sink H, Whitington PM (1991) Location and connectivity of abdominal motoneurons in the embryo and larva of Drosophila melanogaster. J Neurobiol 22:298-311.

Smith SJ (1988) Neuronal cytomechanics: the actin-based motility of growth cones. Science 242:708-715.

Snow PM, Bieber AJ, Goodman CS (1989) Fasciclin III: a novel homophilic adhesion molecule in Drosophila. Cell 59:313-323.

Sun Y-A, Poo M (1987) Evoked release of acetylcholine form the growing embryonic neuron. Proc Natl Acad Sci USA 84:2540-2544.

Taghert PH, Bastiani MJ, Ho RK, Goodman CS (1982) Guidance of pioneer growth cones: filopodial contacts and coupling revealed with an antibody to Lucifer yellow. Dev Biol 94:391-399.

Thomas JB, Bastiani MJ, Bate M, Goodman CS (1984) From grasshopper to Drosophila: a common plan for neuronal development. Nature 310:203-207. 